\title{
No-Regret Matching Game Algorithm for NOMA Based UAV-Assisted NB-IoT Systems
}

\author{
Samar Shaker Metwaly ${ }^{1 *}$, Ahmed. M. Abd El-Haleem², Osama El-Ghandour ${ }^{2}$ \\ ${ }^{1}$ Electronic and Communication Department, Misr University for Science and Technology, Cairo 11795, Egypt \\ ${ }^{2}$ Electronic and Communication Department, Helwan University, Cairo 11795, Egypt
}

Corresponding Author Email: samarshaker2904@ gmail.com

https://doi.org/10.18280/isi.260108

Received: 28 November 2020

Accepted: 3 February 2021

\section{Keywords:}

NB-IoT, UAV, regret matching, NOMA, matching game, LTE, URLLC, mMTC

\begin{abstract}
NB-IoT is the standardized technology for machine type communication (MTC) in Long Term Evolution (LTE). NB-IoT can achieve IoT requirements nevertheless, it suffers a low rate and capacity. On the other hand, Unmanned aerial vehicles (UAV) and Non-Orthogonal Multiple Access (NOMA) are promising technology used to enhance the throughput, capacity, and coverage of wireless communication networks. In this paper, we propose a heterogeneous network scenario where a UAV small Base Station (UBS) is used to assist the LTE Macro Base Station (MBS) with the help of the Non-Orthogonal Multiple Access technique to solve the NB-IoT throughput and capacity issues. Matching game based noregret learning algorithm is proposed to optimize the NB-IoT device association and using NOMA pairing at each base station to provide the maximum system total rate and capacity. Simulation results show that our proposed scheme increases the total rate of the system by $60 \%$ and the system capacity by at least $80 \%$, compared to NOMA without UAV and the total rate and capacity of the system by $200 \%$ and $85 \%$ respectively, with OMA scheme.
\end{abstract}

\section{INTRODUCTION}

Internet of Things is a promising technology that aims to change different aspects of our life. IoT interconnects the physical objects so a massive connected and extend coverage is required [1]. On the other hand, $4 \mathrm{G}$ has been widely used in IoT that some techniques are introduced for IoT as NB-IoT and eMTC. International Telecommunications Union (ITU) classifies the IoT devices according to its Quality of Service (QoS) requirements into massive machine-type communications (mMTC) and ultra-reliable and low latency communications (URLLCs) in LTE [2].

3GPP has introduced the narrowband internet of things (NB-IoT) in its recent release 13 as a technology that can satisfy most of IoT requirements. Nevertheless, NB-IoT suffers low rate, capacity as a result of its limited bandwidth $(180 \mathrm{KHz})$ so one of the important issues in NB-IoT is the efficient usage of its resources to support massive NB-IoT devices [3, 4]. NB-IoT provides a downlink data rate of $250 \mathrm{~kb} / \mathrm{s}$ and an uplink data rate of $15 \mathrm{~kb} / \mathrm{so}$, many researchers interests in how to enhance the rate of the system. On the other hand, a promising technology called Unmanned aerial vehicle (UAV) uses a flying platform or drone as a part of the wireless network to enhance the communication services to macro cell targets in the case of overload situations. UAV can be used to enhance coverage, throughput and capacity of wireless networks $[5,6]$. Also, NOMA technique has an important role in efficiently using the spectrum to enhance the system capacity by sharing devices the same resources [7]. So, using UAV Base Station (UBS) with the Non-Orthogonal Multiple access (NOMA) technique can help significantly enhances the total throughput and capacity of the NB-IoT system.

\subsection{Related work}

Recently, NB-IoT is considered as one of the important techniques that can satisfy IoT requirements, so more researching papers try to enhance its performance by maximizing the throughput and capacity of NB-IoT system, which has limited bandwidth. UAV is a promising technology that can be used as a flying base station to enhance the performance of the terrestrial system so many researchers using it as a flying drone to enhance the IoT system performance.

In ref. [8] author tries to maximize the rate by introducing a sub-optimal iterative algorithm and cooperative approaches for NB-IoT uplink and downlink, where a QoS-aware resource allocation algorithm is used to minimize the repetition factor of the system. The proposed cooperative approaches are done between the neighbor cells by optimizing the transmitted power of neighbor cells to minimize the interference on each other these are causing maximization in the rate of NB-IoT.

As we mention that the Non-Orthogonal Multiple access (NOMA) is used to enhance the capacity of systems so some researchers use it to enhance the capacity of NB-IoT system [9-13]. Where in ref. [9] the author discusses the fundamentals of uplink and downlink NOMA transmission, then uses NOMA to enhance the connectivity of massive Machin type communication devices (MTCDs) and compared it to conventional orthogonal multiple access.

In ref. $[10,11]$ author proposed a power domain uplink the Non-Orthogonal Multiple access (NOMA) scheme for NBIoT system where, formulate a joint subcarrier and transmission power allocation problems to maximize the number of MTCDs by decomposing the problem into two subproblems and proposed algorithms to solve it so, these are not simple and consume more time. In ref. [12] authors proposed 
a NOMA scheme for the NB-IoT system where uses a clustering method (water filling) that each cluster devices share the same frequency resource considering the required QoS to maximize the total rate of the system. In ref. [13] author provides the Non-Orthogonal Multiple access (NOMA) scheme-based matching game for uplink in NB-IoT systems to maximize the total system rate where the matching game provides an optimizing selection of devices that share the same frequency resource considering required QoS.

On the other hand, some researchers try to benefit the use of UAV as a base station to enhance the capacity and throughput of the wireless network. Where in ref. [14] authors use UAVs as intermediate nodes between the macro and small cell tiers for improving coverage and boosting capacity. Also, in ref. [15] the authors consider UAV-enabled downlink wireless system where the UAV using as a flying base station moves in a circular trajectory around the center of a microcell to provide coverage to the macro user located outside an offloaded BS coverage region and using the Non-Orthogonal Multiple access (NOMA) scheme to enhance the capacity of the system.

In ref. [16] authors try to use UAVs in IoT system to help achieve the IoT requirements, they also analyze the UAV-IoT device associations to provides connections with low transmitting power using combines notions from a many-toone matching game algorithm which help in associate the suitable UAV and no-regret learning algorithm which is used to determine each IoT device's regret and take a decision of better UAV that associates it. OMA scheme is used to communicate the IoT devices.

\subsection{Contribution}

In our paper, we aim to enhance the total rate and capacity of the uplink NB-IoT system by using UBS and NOMA scheme to serve devices on the Macro Base Station (MBS) cell edge that suffers from low coverage and bad channel conditions. In our proposed algorithm we use a many-to-one matching to enables the NB-IoT devices to associate with the base stations which maximizes the overall system rate, then a no-regret learning method is used to improve the association problem and increases the system performance. In the second phase of the proposed algorithm, each base station (MBS or UBS) uses a one-to-one matching game to provide optimium pairing-based NOMA of the NB-IoT devices considering different required rate (URLLC and mMTC). So, the contribution of our paper is

1) This paper proposes using UAV as a base station to enhance the NB-IoT capacity and throughput by allocating on the cell edge which, also improves the cell coverage by serving the cell edge devices. In addition of that, NOMA scheme is used to efficiently utilize the spectrum of the system that, improves the capacity and throughput of NB-IoT.

2) We formulate the optimization problem to maximize the uplink NB-IoT system rate.

3) Matching game with No-regret learning is applied with its two phases to solve our optimization problem which maximizes the total system rate and enhance the capacity of the system.

4) Each base station uses the NOMA scheme for NBIoT devices considering different QoS requirements to increase the capacity of the system.

Our paper contributes greatly to solving the problems of uplink NB-IoT system and can overcome the results of other researching papers for NB-IoT system.

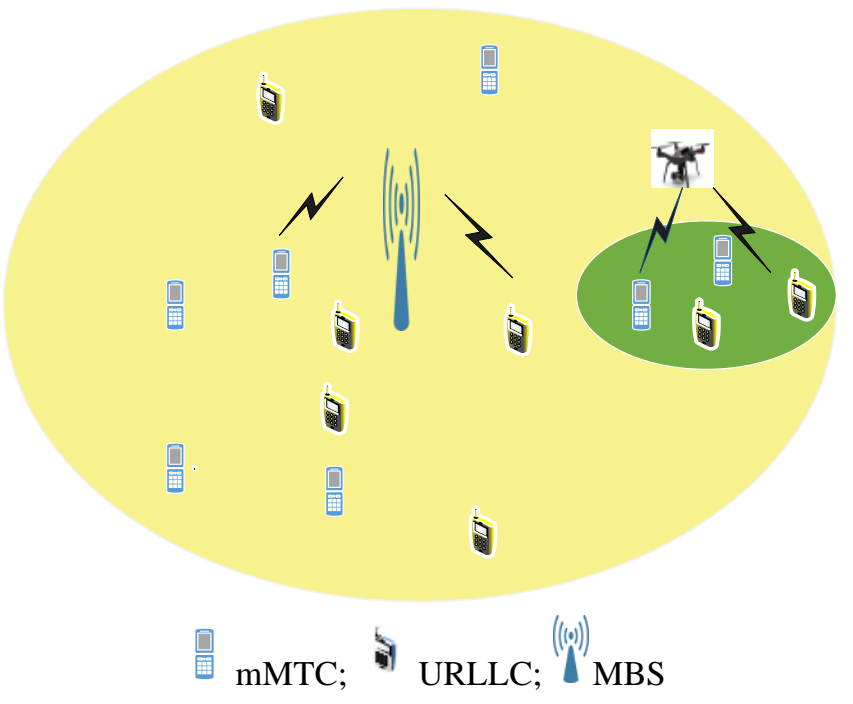

Figure 1. NB-IoT with UAV system model

The rest of this paper is organized as follows. Section II presents the system model of MBS and UBS including NBIoT devices with different required QoS. section III presents the formula of the throughput maximization problem of the system. section IV explains the matching game and NO-regret learning algorithms. In section $\mathrm{V}$ we explain the proposed algorithm. section VI presents the simulation results. Finally, conclude this paper in section VII.

\section{SYSTEM MODEL}

Consider a macro cell base-station with an underlay UBS to enhance its coverage and capacity for the NB-IoT device services on the macro cell edge as shown in Figure 1. The NBIoT devices are distributed randomly under the coverage area with different QoS requirements. According to the ITU definition the NB-IoT devices are classified as URLLC and mMTC. Where each device is associated with the base station (UBS or MBS) which provides it with the required rate with a minimum number of subcarriers. Then, each base station pairs every two users using the Non-Orthogonal Multiple access (NOMA) scheme to increase the system capacity and overall system throughput.

Assume UAV provide wireless coverage radius $R_{u}$ and altitude $h_{u}$, and its locations in the free space can be represented as $\left(x_{U}, y_{U}, h_{U}\right)$. Also, for any NB-IoT device $i$ from the set of the NB-IoT devices $I=\{1,2 \ldots, i, \ldots K\}$, its location can be represented as $\left(x_{i}, y_{i}, z_{i}\right)$. Where the communication channel between NB-IoT devices and UAV base station can be modeled as a Line of sight (LOS) path, and the path loss model can be got by calculating the probability of LOS link from NB-IoT user $i \in I$ towards the UAV base station $U$ through ground-to-air communications channel [16].

$$
p_{i U}^{L O S}=\frac{1}{1+a \exp \left(-b\left[\theta_{i U}-a\right]\right)}
$$

where, $a, b$ are constant in the urban and rural environment, and $\theta_{i U}$ is the elevation angle which equals:

$$
\theta_{i U}=\frac{180}{\pi} \times \sin ^{-1}\left(\frac{h_{U}}{D_{i U}}\right)
$$

Also, $D_{i U}$ is the distance between the NB-IoT user $i$ and the 
associated UAV base station $U$.

$$
D_{i U}=\sqrt{\left(x_{i}-x_{U}\right)^{2}+\left(y_{i}-y_{U}\right)^{2}+\left(z_{i}-h_{U}\right)^{2}}
$$

So, the average path loss can be calculated as:

$$
P L_{i U}=p_{i U}^{L O S} L_{i U}^{L O S}+p_{i U}^{N L O S} L_{i U}^{N L O S}
$$

where, $p_{i U}^{L O S}$ is the probability of not having LOS, $p_{i U}^{N L O S}=$ $1-p_{i U}^{L O S}$. The path loss of line of sight can be calculated as:

$$
L_{i U}^{L O S}=\mu_{1}\left(\frac{4 \pi f_{c} D_{i U}}{c}\right)^{\beta}
$$

and the path loss of not having a line of sight is

$$
L_{i U}^{N L O S}=\mu_{2}\left(\frac{4 \pi f_{c} D_{i U}}{c}\right)^{\beta}
$$

where, $\mu_{1}$ and $\mu_{2}$ are the extreme path loss coefficients in LoS and NLoS, $\boldsymbol{\beta}$ is the exponent of the path loss. Finally, the gain of the air-ground channel is calculated as

$$
G_{i U}=1 / P L_{i U}
$$

On the other hand, the MBS path loss model can be obtained by the standard LTE model which is based on the distance between them(d) as $[17,18]$.

$$
P L_{j M}=128.1+37.6 \log (d)
$$

where, $\mathrm{d}$ is the distance between the NB-IoT device and the macro-base station. The gain of the MBS channel $G_{j M}=$ $1 / P L_{j M}$.

Finally, the signal to interference noise ratio SINR of the uplink communication channel between the NB-IoT device $i$ and UAV base station can be calculated as,

$$
\gamma_{i U}=\frac{P_{i U} G_{i U}}{P_{j M} G_{j U}+\sigma^{2}}
$$

where $P_{i U}$ is the transmitted power from NB-IoT device $i$, $G_{i U}$ is the channel gain, and $\sigma^{2}$ is the noise power. The interference from the MBS NB-IoT device can be presented by $P_{j M} G_{j U}$, where $P_{j M}$ is the MBS NB-IoT device $j$ transmitted power, and $G_{j U}$ is the channel gain between the interfering device and the UAV base station.

Likewise, the signal to interference noise ratio SINR of the uplink channel between the NB-IoT device $j$ and its associated MBS can be calculated as,

$$
\gamma_{j M}=\frac{P_{j M} G_{j M}}{P_{i U} G_{i M}+\sigma^{2}}
$$

where, $P_{j M}$ is the uplink transmitted power from NB-IoT device $j, G_{j M}$ is the channel gain, and the interference from the UBS NB-IoT device is $P_{i U} G_{i M}$.

According to the adaptive modulation and coding scheme (MCS) used in LTE, the actual uplink data rate of any NB-IoT device can be calculated physically depending on mapping the corresponding SINR [18] as,

$$
R^{p h}=\frac{N_{f B} N_{s u} \cdot N_{n} \cdot N_{b x} \cdot E C R_{x}}{T_{S f}} \times \frac{1}{R_{e p x}}
$$

where, $N_{f B}$ is the number of frequency blocks in bandwidth $B$, $N_{\text {su }}$ is the number of subcarriers in one Block, $N_{n}$ is the number of symbols on each subcarrier, $T_{S f}$ is the duration of one subframe, $R_{e p x}$ is the repetition factor. $N_{b x}$ is the number of bits in each symbol and $E C R_{x}$ is the effective code rate, which can be determined from the Channel Quality Indicator (CQI) index, which depending on the SINR value.

\section{PROBLEM FORMULATION}

In this section, the system maximization problem is formulated to maximize the overall system throughput, which is done in two steps. The first step is the association of the NBIoT device to one base station MBS or the UBS, then the second step is the pairing between every two users using the Non-Orthogonal Multiple access (NOMA) scheme at each base station. So, the maximization problem can be formulated as:

$$
\text { P: } \max \sum_{i \in \mathrm{U}} \sum_{i \in \mathrm{G}} X_{i B} R_{i}^{p h}
$$

Subject to

$$
\begin{gathered}
\mathrm{C} 1: \gamma_{i U}>\gamma_{U t h} \text { or } \gamma_{i M}>\gamma_{M t h}, \forall i \in \mathrm{I} \\
\mathrm{C} 2: P_{r i}>P_{r j}, \forall i \in \mathcal{M} \text { and } \mathrm{j} \in \mathcal{N} \\
\mathrm{C} 3: P_{t i} \text { and } P_{t j} \leq P_{\max }, \forall i \text { and } j \in \mathrm{I} \\
\mathrm{C} 4: X_{i B}=\{0,1\}
\end{gathered}
$$

The constrain $\mathrm{C} 1$ is to assure that the NB-IoT device is under the coverage of the UBS and/or the MBS. Constrain C2 is to achieve the NOMA pairing condition, where each base station classifies its NB-IoT devices according to the received power $P_{r i}$ into one of the two groups, group $\mathcal{M}$ contains NBIoT devices with high received power at the base station and group $\mathcal{N}$ contains NB-IoT devices with low received power at the base station. So, constrain $\mathrm{C} 2$ is to assure that the pairing is done under the condition of the NOMA scheme to achieve the perfect successive interference cancellation (SIC). condition. Constrain $\mathrm{C} 3$ means that the transmitted power $P_{t i}$ of the NB-IoT device $i$ be smaller than the maximum transmitted power. Finally, constrain $\mathrm{C} 4$ indicates that the association indicator which takes a binary value.

\section{MATCHING GAME ALGORITHM}

The matching game is used to solve the maximization problem with enhanced performance using a low complexity algorithm. In this paper, we apply a many-to-one matching game to associate NB-IoT devices to the base station MBS or UBS which Provides the required rate for each NB-IoT device by using a minimum number of resources to enhance the total rate of the system. Also, each base station applies one-to-one matching to optimize the pairing of NB-IoT devices in the used the Non-Orthogonal Multiple access (NOMA) scheme to increase the total rate and the capacity of the system. 


\subsection{Cell base station association}

In this type of matching game $\Phi$, many agents from one side of matching are matched with one agent on the other side. Where the many-to-one matching function $[19,20]$. In our proposed technique the NB-IoT devices are one side of matching and the MBS and UBS are the other side, where each NB-IoT device select and send an association request to a base station depending on the NB-IoT device preference list which calculated using its utility function $U_{t i}$. Then, each base station accepts or reject this request according to its preferences calculated using the utility function $U_{t B}$, and its quota $q$.

The NB-IoT utility function $U_{t i}$ can be calculated as

$$
U_{t i}=N_{s c}^{-1}
$$

where, the NB-IoT device preferred to associate the base station that achieves its required rate with a minimum number of resources $N_{s c}$, which is calculated as

$$
N_{s c}=\frac{R_{e q}}{R^{p h}}
$$

where, $R_{e q}$ is the NB-IoT device required rate. Then, each NB-IoT device creates its preference list in ascending order.

The utility function UBS and MBS is to accepts the request for the NB-IoT devices maximums the overall system rate, which calculated as:

$$
U_{t B}=R^{p h}
$$

As we mention, the device may not be accepted from the base station, in this case, the NB-IoT device sends an association request to the next base station in its preference list.

\subsection{NO-regret learning based on matching game}

Regret matching is suitable for machine type communication. It used by many research papers which show that Regret matching learning achieves better performance in term of system throughput more than the random allocation, and converges rapidly to a correlated equilibrium of the system. In addition of that, it allows us to consider the neighbors' actions and to adapt to the environment [16, 21-23].

In the proposed algorithm, no-regret learning algorithm is used to update NB-IoT device choice of base stations association, where it enables NB-IoT devices to associate with a base station which can result a lower regret than the current possible base station regret. This is done depending on calculating the utility function of the NB-IoT device (11) to obtain the optimum choice which enhances the performance of the system [16]. For example, if a device associated the UAV base station which can satisfy the required rate of this device with four resources but when calculating the required resources for the device rate when associated the macro base station MBS it founds that it will use two resource only so, it is more appropriate to transfer this device to associate with the MBS instead of the UAV base station, which will enhance the total rate and capacity of the system and converges rapidly to its correlated equilibrium For this reason, we use the no-regret algorithm.

Assuming $\rho_{i}^{t}$ is the regret of NB-IoT device $i$ at time $t$ after choosing an action $\alpha_{i}^{a}$ instead of the action $\alpha_{i}^{b}$ in the previous iteration $t-1$.

$$
\begin{aligned}
& \rho_{\mathrm{i}}^{\mathrm{t}}\left(\alpha_{\mathrm{i}}^{\mathrm{a}}, \alpha_{\mathrm{i}}^{\mathrm{b}}\right) \triangleq \max \left\{D_{i}^{t}\left(\alpha_{\mathrm{i}}^{\mathrm{a}}, \alpha_{\mathrm{i}}^{\mathrm{b}}\right), 0\right\} \\
& D_{i}^{t}\left(\alpha_{\mathrm{i}}^{\mathrm{a}}, \alpha_{\mathrm{i}}^{\mathrm{b}}\right) \\
& =\frac{1}{T} \sum_{t \leq T}\left(\mathrm{Ut}_{\mathrm{i}}^{\mathrm{t}}\left(\alpha_{\mathrm{i}}^{\mathrm{b}}, \alpha_{-i}\right)_{-} \mathrm{Ut}_{\mathrm{i}}^{\mathrm{t}}\left(\alpha_{\mathrm{i}}^{\mathrm{a}}, \alpha_{-i}\right)\right)
\end{aligned}
$$

where, $U t_{i}^{t}\left(\alpha_{i}^{a}, \alpha_{-i}\right)$ is the utility function of NB-IoT device $i$ at time $t . D_{i}^{t}\left(\alpha_{i}^{a}, \alpha_{i}^{b}\right)$ is the average regret that the device $i$ will get if it makes the action $\alpha_{i}^{b}$ instead of the action $\alpha_{i}^{a}$. So, if $D_{i}^{t}\left(\alpha_{i}^{a}, \alpha_{i}^{b}\right)>0$ this means the device $i$ will have regret if make the action $\alpha_{i}^{b}$ than $\alpha_{i}^{a}$. In our case this mean the device $i$ can achieve its required rate by a smaller number of resources (its utility function) if stays in this base station and doesn't take any transfer action. if $D_{i}^{t}\left(\alpha_{i}^{\mathrm{a}}, \alpha_{\mathrm{i}}^{\mathrm{b}}\right) \leq 0$ this means that the device $i$ will not have any regret if make different action than $\alpha_{i}^{a}$ ( transfer to another base station). So, in our proposed technique device $i$ check the $\rho_{i}^{t}\left(\alpha_{i}^{a}, \alpha_{i}^{b}\right)$ in each iteration to decide if stays this associated base station or change the association.

\subsection{NOMA pairing scheme}

The one-to-one matching game is used to achieve optimized pairing of devices, based on the Non-Orthogonal Multiple access (NOMA) scheme where the one-to-one matching game algorithm is done between two agents each on a side where each agent on a side implements his preference list according to its utility function. the one-to-one matching game is defined as $\mu_{D P}: \mathrm{M} \rightarrow \mathrm{N}$, where $\mu_{D P}$ is the outcome of matched devices $[19,20]$, where each base station divides its associated devices into two groups $\mathrm{M}, \mathrm{N}$ for pairing where the device $m \in \mathcal{M}$ utility function can be defined as:

$$
U_{m}=\gamma_{m}, \quad \forall m \in \mathcal{M} \text { and } n \in \mathcal{N}
$$

Each $m \in \mathcal{M}$ device maximizing its SINR by minimizing the interference caused due to the pairing with $n \in \mathcal{N}$ device. The device $n \in \mathcal{N}$ utility function can be defined as:

$$
U_{n}=R_{m, n}^{p h}, \quad \forall m \in \mathcal{M} \text { and } n \in \mathcal{N}
$$

Also, each $n \in \mathcal{N}$ device pairs with a device $m$ that maximizing the total rate.

\section{THE PROPOSED ALGORITHM}

In our proposed algorithm initially, each NB-IoT device calculates its utility and implements its preference list based on (9). Then it sends a request to the neighbor base stations UBS or the MBS, each base station accepts or rejects the requested device based on (12) and repeats until achieve stable matching $\Phi$. Then apply the no- regret matching of each device based on (14), (15) to obtain the no regret association of base station for each NB-IoT deice. After the association is done each base station applies a one-to-one matching game algorithm based on the Non-Orthogonal Multiple access (NOMA) technique where determines the required subcarriers of each device according to its required rate (URLLC or mMTC). Then it optimally selects devices for NOMA pairing depending one-to-one matching game. 


\section{Algorithm 1: Matching game for NB-IoT Devices}

- Initialization: Discover each NB-IoT device's location coordinates.

- Initialization: URLLC, mMTC groups according to the required rate.

- Each device discovers its neighbor base stations and calculates the channel gain with them.

- Many-to-one matching game

-Each NB-IoT device implement its preference list according to its utility function on (11) and send a request to base stations (UBS or MBS).

-Each base station (UBS or MBS) implements its preference list according to its utility function on (13) where decide accept or reject each device request.

-repeat

After accepted NB-IoT devices associated with its preferred base station, the rejected devices re-apply to their next best choice.

-Until

Each NB-IoT device associates a base station. having a stable matching $\Phi$.

\section{- No-Regret Matching game}

After the association is done between each NB-IoT device $i$ and the base stations $\left(\alpha_{i}\right)$.

-Each device checks the regret $\rho_{i}^{t}\left(\alpha_{i}^{a}, \alpha_{i}^{b}\right)$ to decide stays in the current associated base station or transfers to another base station depending on its utility function $\mathrm{Ut}_{\mathrm{i}}(11)$

-update the selection of the base station according to the regret at $\mathrm{t}+1$

- Each base station applies the one-to-one matching game

-Determine the required subcarrier for each device. -Classify $\mathcal{M}, \mathcal{N}$ groups.

1- Every device $n \in \mathcal{N}\left(d_{n i}\right)$ construct a preference list ( $L_{n i}$ ) using $U_{m}$

2- $\quad$ While $\Sigma \forall \mathrm{i}, j Q_{j \rightarrow i} \neq 0$ do:

3- For each unpaired device $n \in \mathcal{N}$

Find $L_{n i}$

4- $\quad$ Select the preferred device from the list $L_{n i}$

5- Send a request $Q_{j \rightarrow i}$ of each device $n \in \mathcal{N}$.

6- For each device, $m \in \mathcal{M}$ construct preference $\operatorname{list}\left(L_{m j}\right)$ using $U_{n}$ according to $Q_{j \rightarrow i}$

7- Accept $d_{n i}$ from $L_{m j}$ and reject the unpreferred.

8- Repeat

9- end while

10- Calculate $R_{T}^{p h}$

11- Results: a stable matching $\mu_{\mathrm{DP}}$

- calculate the summation of the system rate.

\section{SIMULATION RESULTS}

In this section, we introduce the performance evaluation of our proposed Algorithm, by comparing it with the NB-IoTbased NOMA matching game algorithm in ref. [13] and the UAV with the matching game and NO-Regret based OMA technique in ref. [16]. In the simulation, we consider one macro cell base station with an underlying UAV base station at its edge and randomly uniform distribution NB-IoT devices (URLLC, mMTC) over its area. The simulation parameters are shown in Table 1.

In Figure 2 shown the overall system rate variation versus the number of NB-IoT devices. we observe that our proposed algorithm maximizing the overall system rate compared to the other algorithms (UAV with OMA based regret matching, and NOMA without UAV). Also, the figure introduces a comparison between our proposed algorithm which using the no-regret algorithm and without using no-regret algorithm. This comparison illustrates that the no-regret learning enhances the total rate of the system which is increased by approximately $12 \%$ than algorithm without no-regret.

Table 1. Simulation parameters

\begin{tabular}{cc}
\hline Simulation parameters & Value \\
\hline The Cell radius & $2 \mathrm{~km}$ \\
UAV altitude & $150 \mathrm{~m}$ \\
UAV radius & $200 \mathrm{~m}$ \\
NB-IoT bandwidth & $180 \mathrm{KHz}$ \\
Number of subcarriers for & 48 subcarriers \\
3.75KHZ/subcarrier & $-174 \mathrm{dBm} / \mathrm{HZ}$ \\
Additive white Gaussian noise & $23 \mathrm{dBm}$ \\
Max transmit power $\left(P_{\text {max }}\right)$ of NB-IoT & $2-20 \mathrm{~kb} / \mathrm{s}$ \\
The required rate of URLLC devices & $0.1-2 \mathrm{~kb} / \mathrm{s}$ \\
The Threshold rate of mMTC devices & $1 \mathrm{block}$ \\
The number of frequency blocks in & $(\mathrm{a}, \mathrm{b})=$ \\
bandwidth B & $(11.95,0.14)$ \\
Constant values in Urban environment $(\mathrm{a}, \mathrm{b})$ &
\end{tabular}

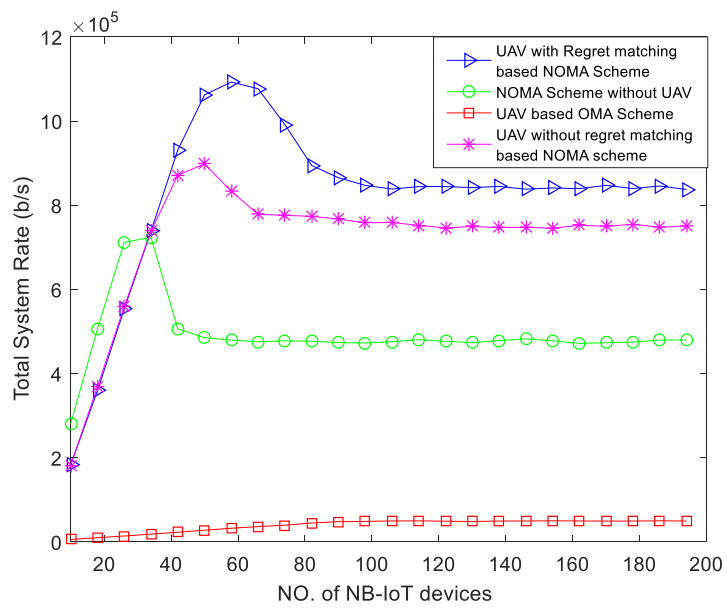

Figure 2. Total rate versus the number of NB-IoT devices

In addition, we can see from the figure that at a small number of NB-IoT devices our proposed scheme provides a system rate approximately the same with the NOMA without UAV. Where the effect of UAV in providing better rate for the device on cell edge appearing more with increasing the number of devices. In general, our proposed scheme success to enhance the total rate on the average by $60 \%$ above the NOMA without UAV and $200 \%$ above UAV with OMA technique.

As shown in Figure 3. we can see that our proposed scheme enhances the capacity of the system versus at different values of the NB-IoT devices required rate at a constant number of devices. As we can see our proposed algorithm increases the capacity significantly compared to the other algorithms where it enhances the capacity by $100 \%$ above the NOMA-without UAV and $110 \%$ above the UAV with OMA technique for NBIoT devices. On the other hand, our proposed scheme with noregret learning enhances the system capacity by $10 \%$ at low average required rate than without using no-regret learning.

Figure 4 illustrates that the capacity of the NB-IoT system with respect to a different number of NB-IoT devices at a 
constant required rate for URLLC and mMTC devices. We observe that our proposed algorithm also successful in enhancing the capacity by increasing the number of devices than the NOMA without UAV and UAV with OMA algorithms as a result of optimal association which reduces the number of the required subcarrier of each device so it enables the system to serve more NB-IoT devices. Also, our proposed algorithm comparing with using UAV with OMA technique overcomes by $85 \%$ because NOMA technique improves the spectrum efficiency which allows serving more devices so increase the capacity as shown. However, the figure shows that the no-regret algorithm slightly can enhance the capacity than the other without using no-regret algorithm.

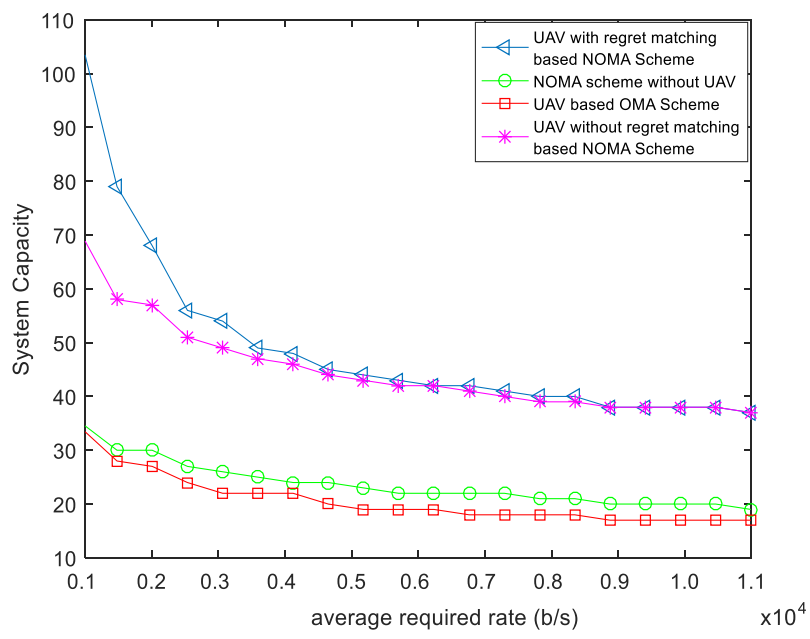

Figure 3. System capacity versus the average required rate of NB-IoT devices

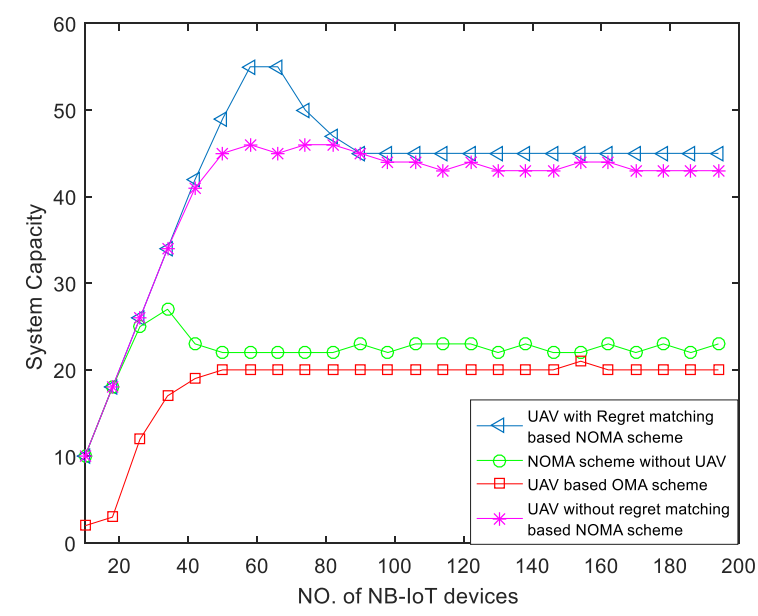

Figure 4. System capacity versus the number of NB-IoT devices

\section{CONCLUSION}

In this paper, we proposed an Unmanned aerial vehicle as a base station to provide coverage to devices on the macro cell edge based on the Non-Orthogonal Multiple access (NOMA) scheme for uplink NB-IoT system that enhances the capacity of the system. We formulate a problem to maximize the total throughput of the system. Then, we analyze the association of the NB-IoT device and propose many-to-one matching game and no-regret learning algorithms to obtain an optimized association of base station (MBS or UAV). Each base station pairs its NB-IoT devices using the NOMA scheme over the same resources based on the proposed matching game algorithm. The proposed algorithm success in NB-IoT issues and the simulation results show that our proposed scheme overcomes NOMA without UAV scheme and the UAV with OMA for NB-IoT throughput by $70 \%$ and $200 \%$. Also, the capacity of our proposed scheme overcomes by $100 \%$ with NOMA without UAV scheme and $85 \%$ with UAV with OMA scheme. Using no-regret learning in our proposed algorithm enhances the total system rate and have a little bit effect on the system capacity.

\section{REFERENCES}

[1] Chafii, M., Bader, F., Palicot, J. (2018). Enhancing coverage in narrow band-IoT using machine learning. In 2018 IEEE Wireless Communications and Networking Conference (WCNC), pp. 1-6. https://doi.org/10.1109/WCNC.2018.8377263

[2] Li, S., Da Xu, L., Zhao, S. (2018). 5G internet of things: a survey. Journal of Industrial Information Integration, 10: 1-9. https://doi.org/10.1016/j.jii.2018.01.005

[3] Chen, M., Miao, Y., Hao, Y., Hwang, K. (2017). Narrow band internet of things. IEEE Access, 5: 20557-20577. https://doi.org/10.1109/ACCESS.2017.2751586

[4] Malik, H., Pervaiz, H., Alam, M.M., Le Moullec, Y., Kuusik, A., Imran, M.A. (2018). Radio resource management scheme in NB-IoT systems. IEEE Access, 6: 15051-15064. https://doi.org/10.1109/ACCESS.2018.2812299

[5] Li, B., Fei, Z., Zhang, Y. (2018). UAV communications for $5 \mathrm{G}$ and beyond: Recent advances and future trends. IEEE Internet of Things Journal, 6(2): 2241-2263. https://doi.org/10.1109/JIOT.2018.2887086

[6] Mozaffari, M., Saad, W., Bennis, M., Nam, Y.H., Debbah, M. (2019). A tutorial on UAVs for wireless networks: Applications, challenges, and open problems. IEEE Communications Surveys \& Tutorials, 21(3): 2334-2360. https://doi.org/10.1109/COMST.2019.2902862

[7] Chen, M., Miao, Y., Hao, Y., Hwang, K. (2017). Narrow band internet of things. IEEE Access, 5: 20557-20577. https://doi.org/10.1109/ACCESS.2017.2751586

[8] Malik, H., Pervaiz, H., Alam, M.M., Le Moullec, Y., Kuusik, A., Imran, M.A. (2018). Radio resource management scheme in NB-IoT systems. IEEE Access, 6:

15051-15064. https://doi.org/10.1109/ACCESS.2018.2812299

[9] Tabassum, H., Ali, M.S., Hossain, E., Hossain, M., Kim, D.I. (2016). Non-orthogonal multiple access (NOMA) in cellular uplink and downlink: Challenges and enabling techniques. arXiv preprint arXiv:1608.05783.

[10] Mostafa, A.E., Zhou, Y., Wong, V.W. (2017). Connectivity maximization for narrowband IoT systems with NOMA. In 2017 IEEE International Conference on Communications (ICC), pp. 1-6. https://doi.org/10.1109/ICC.2017.7996362

[11] Li, S., Wang, Q., Sun, Y., Cui, X. (2018). Resource management for non-orthogonal multiple access based machine type communications. In Proceedings, APSIPA Annual Summit and Conference, 201: 413-419.

[12] Shahini, A., Ansari, N. (2019). NOMA aided narrowband IoT for machine type communications with 
user clustering. IEEE Internet of Things Journal, 6(4): 7183-7191. https://doi.org/10.1109/JIOT.2019.2914947

[13] Metwaly, S.S., Abd El-Haleem, A.M., El-Ghandour, O. (2020). NOMA based matching game algorithm for narrowband internet of things (NB-IoT) system. Ingénierie des Systèmes d'Information, 25(3): 345-350. https://doi.org/10.18280/isi.250308

[14] Sharma, V., Bennis, M., Kumar, R. (2016). UAVassisted heterogeneous networks for capacity enhancement. IEEE Communications Letters, 20(6): 1207-1210. https://doi.org/10.1109/LCOMM.2016.2553103

[15] Sharma, P.K., Kim, D.I. (2017). UAV-enabled downlink wireless system with non-orthogonal multiple access. In 2017 IEEE Globecom Workshops (GC Wkshps), 1-6. https://doi.org/10.1109/GLOCOMW.2017.8269066

[16] Lhazmir, S., Oualhaj, O.A., Kobbane, A., Ben-Othman, J. (2020). Matching game with no-regret learning for IoT energy-efficient associations with UAV. IEEE Transactions on Green Communications and Networking, 4(4): 973-981. https://doi.org/10.1109/TGCN.2020.3008992

[17] Santos, I., Vieira, P., Borralho, R., Queluz, M.P., Rodrigues, A. (2018). Emulating a software defined LTE radio access network towards 5G. In 2018 International Conference on Communications (COMM), pp. 1-376. https://doi.org/10.1109/ICComm.2018.8484764

[18] Anany, M., Elmesalawy, M.M., Abd El-Haleem, A.M. (2019). Matching game-based cell association in multi- rat HetNet considering device requirements. IEEE Internet of Things Journal, 6(6): 9774-9782. https://doi.org/10.1109/JIOT.2019.2931448

[19] Gu, Y., Saad, W., Bennis, M., Debbah, M., Han, Z. (2015). Matching theory for future wireless networks: Fundamentals and applications. IEEE Communications Magazine, 53(5): 52-59. https://doi.org/10.1109/MCOM.2015.7105641

[20] An, C., Liu, Y. (2011). A matching game algorithm for spectrum allocation based on POMDP model. In 2011 7th International Conference on Wireless Communications, Networking and Mobile Computing, pp. 1-3. https://doi.org/10.1109/wicom.2011.6036726

[21] Greenwald, A., Jafari, A. (2003). A general class of noregret learning algorithms and game-theoretic equilibria. In Learning Theory and Kernel Machines, 2-12. https://doi.org/10.1007/978-3-540-45167-9_2

[22] Lhazmir, S., Kobbane, A., Ben-Othman, J. (2018). Channel assignment for D2D communication: A regret matching based approach. In 2018 14th International Wireless Communications \& Mobile Computing Conference (IWCMC), pp. 322-327. https://doi.org/10.1109/IWCMC.2018.8450520

[23] Sroka, P., Kliks, A. (2014). Distributed interference mitigation in two-tier wireless networks using correlated equilibrium and regret-matching learning. In 2014 European Conference on Networks and Communications (EuCNC), pp. 1-5. https://doi.org/10.1109/EuCNC.2014.6882640 